\title{
MicroRNA-150-5p and SRC kinase signaling inhibitor 1 involvement in the pathological development of gastric cancer
}

\author{
XIYUN QUAN ${ }^{1 *}$, DONGLIANG CHEN ${ }^{1 *},{\text { MING } \mathrm{LI}^{2}, \mathrm{XUN} \mathrm{CHEN}^{3} \text { and MEIYUAN HUANG }}^{1}$ \\ Departments of ${ }^{1}$ Pathology, ${ }^{2}$ Emergency and ${ }^{3}$ Hepatic Surgery, Zhuzhou Central Hospital, Zhuzhou, Hunan 412000, P.R. China
}

Received June 28, 2018; Accepted January 10, 2019

DOI: $10.3892 / \mathrm{etm} .2019 .7828$

\begin{abstract}
The current study aimed to assess the regulatory mechanism of microRNA-150-5p (miR-150-5p) in the pathogenesisofgastriccancer.Reversetranscription-quantitative polymerase chain reaction (RT-qPCR) was performed to verify the expression of miR-150-5p in gastric cancer tissues and cell lines, which was revealed to be highly expressed in each. In addition, the expression of miR-150-5p was associated with advanced gastric cancer and lymph node metastasis. The current study then hypothesized that SRC kinase signaling inhibitor 1 (SRCIN1) was the target gene of miR-150-5p, a theory that was confirmed via a dual luciferase reporter gene assay. RT-qPCR and western blotting were then performed to verify the expression of SRCIN1 in gastric cancer tissues and cell lines. The results demonstrated that SRCIN1 was lowly expressed in gastric cancer tissues and cells. To assess the effect of miR-150-5p on gastric cancer cells, experiments were conducted with BGC-823 cells transfected with a miR-150-5p inhibitor or a miR-150-5p inhibitor+SRCIN1-small interfering (si)RNA respectively. A cell counting kit-8 assay and flow cytometry were also used to assess cell viability and apoptosis, respectively. Western blotting and RT-qPCR were further used to measure the expression of specific markers of epithelial mesenchymal transition (EMT), including epithelial cell markers (E-cadherin and zona occluding-1) and interstitial cell markers (vimentin, $\mathrm{N}$-cadherin and $\beta$-catenin). The results revealed that the miR-150-5p inhibitor attenuated cell viability, induced apoptosis, decreased the expression of interstitial cell markers and increased epithelial cell marker expression. However, all effects of the miR-150-5p inhibitor were reversed following SRCIN1-siRNA treatment. In summary, the current study indicated that the miR-150-5p inhibitor attenuated cell
\end{abstract}

Correspondence to: Dr Ming Li, Department of Emergency, Zhuzhou Central Hospital, 116 Changjiang South Road, Zhuzhou, Hunan 412000, P.R. China

E-mail: liming180601@163.com

*Contributed equally

Key words: microRNA-150-5p, SRC kinase signaling inhibitor 1, gastric cancer, BGC-823 cells viability, induced apoptosis and inhibited gastric cancer cell EMT by targeting SRCIN1.

\section{Introduction}

Gastric cancer (GC) is one of the most common malignant tumors of the digestive tract in the world (1). Despite the continuous development of novel treatment options for GC, the prognosis of the disease remains poor (1-3). Many patients with GC exhibit tumor metastasis upon diagnosis, making treatment very difficult $(4,5)$. Therefore, further elucidating the molecular mechanism of GC to determine early diagnostic markers, therapeutic targets, novel molecular targeted therapeutic drugs and indicators of metastasis and recurrence is of great importance in GC.

MicroRNAs (miRNAs) are a type of non-coding small RNA containing 20-24 nucleotides that regulate the expression of their target genes primarily at the post-transcriptional level (6-8). Thereby, miRNAs participate in a variety of cell functions including cell proliferation, apoptosis, differentiation, metabolism and endocrine system regulation $(6,7,9)$. The abnormal expression of miRNA is associated with a variety of diseases including tumors (7). miRNA has been demonstrated to be involved in the regulation of tumor cell proliferation, apoptosis, differentiation, drug resistance, invasion and metastasis, among which miR-150 also ion, drug resistance, invasion and metastasis, among which miR-150 also serves an important role in the development of tumors (10-15). In colorectal cancer, breast cancer and melanoma, miR-150 is significantly downregulated and exerts a tumor suppressive role (11-13). However, in cervical cancer, miR-150 is highly expressed and promotes tumor progression (14). Therefore, miR-150 serves different roles in different tumor types (10-14). miR-150-5p, a member of the miR-150 family, has been determined to inhibit cancer cell aggressiveness by targeting cwcv and kazal like domains proteoglycan 1 in head and neck squamous cell carcinoma (15). miR-150-5p affects cell proliferation, apoptosis and epithelial-mesenchymal transition (EMT) by regulating the BRAFV600E mutation in papillary thyroid cancer cells (16). miR-150-5p has also been identified as a novel prognostic biomarker in non-small cell lung cancer (17). Yu et al (18) hypothesized that the aryl hydrocarbon receptor enhanced the expression of miR-150-5p to suppress cell proliferation and invasion in prostate cancer by regulating MAP3K12. Furthermore, Suetsugu et al (19) revealed that miR-150-5p 
inhibits the aggressiveness of lung squamous cell carcinoma cells. These results indicate the important roles of miR-150-5p in tumor progression. However, to the best of our knowledge, the role of miR-150-5p in the pathological development of GC remains largely unknown.

EMT is an important component of wound healing and stem cell behavior (20), which may develop fibrosis and tumor progression under pathological conditions (20-22). In addition, tumor cells can obtain transfer force and invasiveness via certain signaling pathways, which include the TGF- $\beta / \mathrm{Smad}$ pathway (23). For example, the reorganization of the cytoskeleton and prominent membrane behavior are all key features of tumor invasion $(23,24)$. In order to leave the primary tumor and invade surrounding tissue, tumor cells must destroy contact between cells, recombine the attachment site of the cell-matrix and generate chemotaxis under the guidance of the extracellular matrix $(23,24)$. In EMT, polarized epithelial cells, which originally exhibit no activity, dissolve their own intercellular junctions into independent, depolarizing and active metastatic mesenchymal cells $(22,24)$. For example, the expression and function of E-cadherin, which supports epithelial cell junctions, disappear, while $\mathrm{N}$-cadherin, which supports mesenchymal cell-cell attachment, is induced (21). The expression of $\mathrm{N}$-cadherin triggers cell metastasis and invasion. Vimentin contributes to EMT cancer cell mechanics by mediating cytoskeletal organization and focal adhesion maturation (25). Highly and abnormally expressed $\beta$-catenin, a multifunctional protein that serves an important role in physiological homeostasis, results in various diseases including cancer, which can serve as transcriptional co-regulators and as adaptor proteins for intracellular adhesion (26). Zonula occluden-1 (ZO-1) is a tight junction-associated protein involved in the maintenance and regulation of epithelial barrier function and is often utilized as an indicator of tight junctional barrier function and permeability of various tissues (27). To the best of our knowledge, the effect of miR-150-5p on GC EMT, particularly on the expression of interstitial cell markers (vimentin, $\mathrm{N}$-cadherin and $\beta$-catenin) and epithelial cell markers (E-cadherin and ZO-1) in GC cells remain unclear.

The current study aimed to assess the role and regulation mechanism of miR-150-5p in the pathological process of GC.

\section{Materials and methods}

Clinical samples. A total of $36 \mathrm{GC}$ tissue and paired normal tissue samples $(2 \mathrm{~cm}$ from the tumor lesion) were collected from patients with GC (age range, 43-67 years; male:female, 1:1; Stage I, 12 cases; Stage II, 12 cases; Stage III-IV, 12 cases; lymph node metastases, 18 cases; no lymph node metastasis, 18 cases) who had undergone surgical resection at Zhuzhou Central Hospital (Zhuzhou, China) from March 2015 to March 2017. None of the patients received any radiotherapy or chemotherapy prior to the surgery. The present study was approved by the Ethical Committee of the Zhuzhou Central Hospital (Zhuzhou, China) and written informed consent was obtained from each patient.

Cell culture. The GC cell lines MGC-803, SGC-7901, BGC-823, and the normal gastric epithelial cell line GES-1 (all Shanghai Guandao Bio-engineering Co., Ltd., Shanghai,
China) were cultured in Dulbecco's modified Eagle medium or RPMI-1640 (Gibco; Thermo Fisher Scientific, Inc., Waltham, MA USA) supplemented with $10 \%$ fetal bovine serum (Gibco; Thermo Fisher Scientific, Inc.) and 1\% penicillin/streptomycin (Sigma-Aldrich; Merck KGaA; Darmstadt, Germany). Cells were incubated in a humidified incubator at $37^{\circ} \mathrm{C}$ with $5 \% \mathrm{CO}_{2}$

Dual luciferase reporter assay. TargetScan bioinformatics software (www.targetscan.org/vert_71) was used to predict the targets of miR-150-5p. It was revealed that SRC kinase signaling inhibitor 1 (SRCIN1) was a potential target of miR-150-5p. To confirm this prediction, wild type (WT-SRCIN1) and mutant (MUT-SRCIN1) 3'-untranslated regions of SRCIN1 were cloned into a pmiR-RB-ReportTM dual luciferase reporter gene plasmid vector (Guangzhou RiboBio Co., Ltd., Guangzhou, China). BGC-823 cells were co-transfected with WT-SRCIN1 or MUT-SRCIN1 and miR-150-5p mimic (5'-UCUCCCAACCCUUGUACC AGUG-3') or mimic control (5'-UUCUCCGAACGUGUC ACGUTT-3') using Lipofectamine ${ }^{\circledR} 3000$ (Invitrogen; Thermo Fisher Scientific, Inc.) following the manufacturer's protocol. miR-150-5p mimic and mimic control were synthesized by Shanghai GenePharma Co., Ltd. (Shanghai, China). Following 48-h incubation, luciferase activity was assessed using the dual-luciferase assay system (Promega Corporation, Madison, WI, USA), according to the manufacturer's protocol. Firefly luciferase activity was normalized to Renilla luciferase activity.

Cell transfection. An miR-150-5p inhibitor and its control (inhibitor control) were synthesized by Shanghai GenePharma Co., Ltd. (Shanghai, China). BGC-823 cells were seeded into 6-well plates $\left(1 \times 10^{6}\right.$ cells per well $)$ and cultured at $37^{\circ} \mathrm{C}$ for $24 \mathrm{~h}$. Cells were transfected with $100 \mathrm{nM}$ miR-150-5p inhibitor (5'-CACUGGUACAAGGGUUGGGAGA-3'), $100 \mathrm{nM}$ inhibitor control (5'-CAGUACUUUUGUGUAGUACAA-3'), $10 \mu \mathrm{M}$ control-siRNA (cat no. abx941273), $10 \mu \mathrm{M}$ SRCIN1-siRNA (cat no. abx905269; both Abbexa, Ltd.) or miR-150-5p inhibitor+SRCIN1-siRNA using Lipofectamine ${ }^{\circledR} 3000$ (Invitrogen; Thermo Fisher Scientific, Inc.), according to the manufacturer's protocol. Transfection efficiency was detected following 48-h transfection via reverse transcription-quantitative polymerase chain reaction (RT-qPCR) or/and western blotting. Cells without any treatment were used as the control.

Cell counting kit (CCK)-8 assay. The current study utilized the CCK8 method to detect cell viability. Following 48-h transfection, BGC-823 cell suspension was adjusted to $1 \times 10^{4}$ cells $/ \mathrm{ml}$ and $100 \mu 1$ cell suspension was added to each well of a 96-well plate. Cells were cultured at $37^{\circ} \mathrm{C}$ with $5 \% \mathrm{CO}_{2}$ for $24 \mathrm{~h}$. Subsequently, $10 \mu 1$ CCK- 8 reagent (Sigma-Aldrich; Merck $\mathrm{KGaA}$ ) was added to each well. Absorbance at the wavelength of $450 \mathrm{~nm}$ was measured using an automatic enzyme-linked immune detector following $2 \mathrm{~h}$ of incubation at $37^{\circ} \mathrm{C}$. The experiment was repeated three times.

Flow cytometry. Following 48-h transfection, BGC-823 cells were digested with $0.2 \%$ trypsin, washed with PBS and fixed with $70 \%$ ethanol overnight at $4^{\circ} \mathrm{C}$. The apoptosis condition of cells was detected using the annexin V-Fluorescein 
isothiocyanate (FITC)/propidium iodide (PI) kit (cat no. 70-AP101-100; Hangzhou MultiSciences (Lianke) Biotech Co., Ltd., Hangzhou, China) according to the manufacturer's protocol. Cell apoptosis rate was measured using a FACS Calibur flow cytometer with FlowJo software (version 7.6.1; FlowJo LLC, Ashland, OR, USA). Cells without any treatment were used as the control group. The assay was performed in triplicate.

Western blotting. Cells (MGC-803, SGC-7901, BGC-823 and GES-1) were washed with ice cold PBS. Total protein was extracted from cells using radioimmunoprecipitation assay buffer (Beyotime Institute of Biotechnology, Shanghai, China) at $4^{\circ} \mathrm{C}$ for $1 \mathrm{~h}$. Protein samples were collected via centrifugation at a speed of $12,000 \mathrm{x} \mathrm{g}$ for $5 \mathrm{~min}$ at $4^{\circ} \mathrm{C}$. Total protein was quantified using a bicinchoninic acid protein assay kit (Beyotime Institute of Biotechnology) and $30 \mu \mathrm{g}$ protein/lane was separated via SDS PAGE on a $10 \%$ gel. The separated proteins were transferred onto polyvinylidene difluoride membranes and blocked with 5\% non-fat milk at room temperature for $2 \mathrm{~h}$. The membranes were incubated with primary antibodies against SRCIN1 (1:1,000; cat no. 3757), N-cadherin (1:1,000; cat no. 13116), vimentin (1:1,000; cat no. 12,826), $\beta$-catenin $(1: 1,000$; cat no. 25362), E-cadherin $(1: 1,000$; cat no. 3195), ZO-1 (1:1,000; cat no. 13663) and $\beta$-actin $(1: 1,000$; cat no. 4970; all Cell Signaling Technology, Inc., Danvers, MA, USA) overnight at $4^{\circ} \mathrm{C}$. Membranes were washed four times with PBST. Following primary incubation, membranes were incubated with horseradish peroxidase-conjugated anti-rabbit Immunoglobulin G secondary antibody (cat no. 7074; 1:2,000; Cell Signaling Technology, Inc.) for $2 \mathrm{~h}$ at room temperature. Membranes were washed four times with PBST. Protein bands were visualized using an ECL reagent (EMD Millipore, Billerica, MA, USA). Protein expression was quantified using AlphaView 3.4.0 software (ProteinSimple, San Jose, CA, USA).

RNA isolation and RT-qPCR. Total RNA was extracted from tissue samples and cells (MGC-803, SGC-7901, BGC-823 and GES-1) using the TRIzol ${ }^{\circledR}$ reagent (Invitrogen; Thermo Fisher Scientific, Inc.). RNA concentration was detected

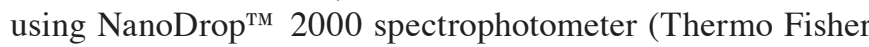
Scientific, Inc.) and samples were stored at $-80^{\circ} \mathrm{C}$ for further use. Total RNA was reverse transcribed into cDNA using the miScript Reverse Transcription kit (Applied Biosystems; Thermo Fisher Scientific, Inc.), according to the manufacturer's protocol. qPCR was subsequently performed using QuantiFast SYBR Green PCR kit (Takara Bio, Inc., Otsu, Japan) using a CFX Connect Real-Time System (Bio-Rad Laboratories, Inc., Hercules, CA, USA). The primer sequences for qPCR were as follows: miR-150-5p forward, 5'-TCGGCGTCTCCC AACCCTTGTAC-3' and reverse, 5'-GTCGTATCCAGTGCA GGGTCCGAGGT-3'; SRCIN1 forward, 5'-AGCCCCGAC AAAAGCAAAC-3' and reverse, 5'-CCAAAGGAAGTC AATACAGGGATAG-3'; N-cadherin forward, 5'-TTTGAT GGAGGTCTCCTAACACC-3' and reverse, 5'-ACGTTTAAC ACGTTGGAAATGTG-3'; vimentin forward, 5'-GACGCC ATCAACACCGAGTT-3' and reverse, 5'-CTTTGTCGTTGG TTAGCTGGT-3'; $\beta$-catenin forward, 5'-AACAGGGTCTGG GACATTAGTC-3' and reverse, 5'-CGAAAGCCAATCAAA CACAAAC-3'; E-cadherin forward, 5'-CGAGAGCTACAC
GTTCACGG-3' and reverse, 5'-GGGTGTCGAGGGAAA AATAGG-3'; ZO-1 forward, 5'-CCTCTGATCATTCCACAC AGTC-3' and reverse, 5'-TAGACATGCGCTCTTCCTCTCT-3'; GAPDH forward, 5'-CTTTGGTATCGTGGAAGGACTC-3' and reverse, 5'-GTAGAGGCAGGGATGATGTTCT-3'; U6 forward, 5'-GCTTCGGCAGCACATATACTAAAAT-3' and reverse, 5'-CGCTTCACGAATTTGCGTGTCAT-3'. The thermocycling conditions were as follows: Initial denaturation at $95^{\circ} \mathrm{C}$ for $10 \mathrm{~min} ; 35$ cycles of $95^{\circ} \mathrm{C}$ for $15 \mathrm{sec}$ and $55^{\circ} \mathrm{C}$ for $40 \mathrm{sec}$. Relative gene expression was calculated using the $2^{-\Delta \Delta \mathrm{Cq}}$ method (28) and U6 and GAPDH served as internal controls for miRNA and mRNA expression, respectively.

Statistical analysis. Statistical analyses were performed using GraphPad Prism 5 software (GraphPad Software, Inc., La Jolla, CA, USA). Data are presented as the mean \pm standard deviation. One-way analysis of variance followed by a Tukey's post-hoc test was used analyze differences among multiple groups and an unpaired or paired Student's t-test was used to analyze the statistical significance between two groups. $\mathrm{P}<0.05$ was considered to indicate a statistically significant result.

\section{Results}

miR-150-5p is highly expressed in GC tissues and cells and is associated with advanced GC and lymph node metastasis. The level of miR-150-5p in GC tissues, paired normal tissues, GC cell lines (MGC-803, SGC-7901 and BGC-823) and the normal gastric epithelial cell line (GES-1) were detected using RT-qPCR. The results demonstrated that compared with normal tissues, miR-150-5p was upregulated in GC tissue (Fig. 1A). Additionally, compared with GES-1 cells, miR-150-5p was significantly upregulated in all GC cell lines and was most abundantly expressed in BGC-823 cells (Fig. 1B). Furthermore, it was revealed that higher levels of miR-150-5p were exhibited in patients with advanced GC, with evidence of lymph node metastases (Fig. 1C). The expression of miR-150-5p gradually increased from Stage I, Stage II, Stage III-IV, non-lymph node metastasis and lymph node metastasis.

SRCIN1 is a target of miR-150-5p, which is lowly expressed in GC tissues and cells. To assess the target genes of miR-150-5p, the current study utilized TargetScan (www.targetscan. org/vert_71). The results demonstrated the existence of binding sites between SRCIN1 and miR-150-5p (Fig. 2A). Additionally, to reveal whether miR-150-5p directly binds to SRCIN1, a dual luciferase reporter assay was performed. The miR-150-5p-SRCIN1-WT or miR-150-5p-SRCIN1-MUT reporter plasmids were co-transfected into BGC-823 cells with miR-150-5p mimics or mimic controls. The results revealed that luciferase activity significantly decreased in the BGC-823 cells co-transfected with miR-150-5p mimic and miR-150-5p-SRCIN1-WT, but not with miR-150-5p-SRCIN1-MUT (Fig. 2B). The data indicates that SRCIN1 is a target gene of miR-150-5p.

It was also demonstrated that the mRNA level of SRCIN1 in GC tissues was lower than that of normal tissues (Fig. 2C). SRCIN1 was also downregulated in all GC cell lines of the current study when compared with GES-1 cells and the expression was lowest in BGC-823 cells (Fig. 2D and E). 

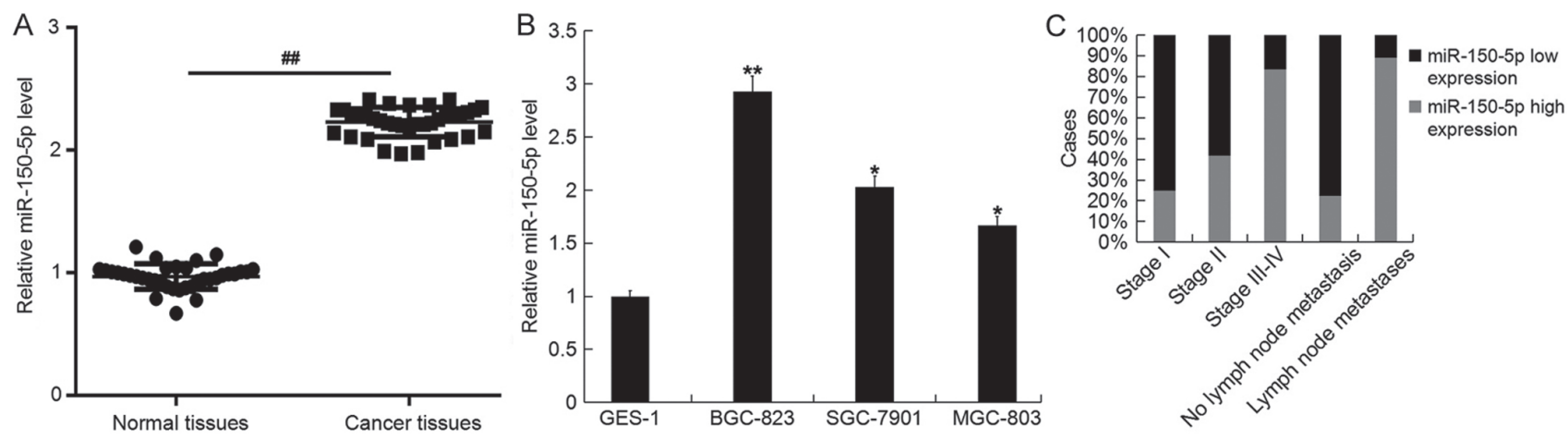

Figure 1. miR-150-5p expression in gastric cancer tissues and cells, and the association between lymph node metastasis and miR-150-5p expression. Reverse transcription-quantitative polymerase chain reaction was performed to detect the level of miR-150-5p. (A) miR-150-5p expression in gastric cancer tissue and paired normal tissue samples. (B) miR-150-5p expression in gastric cancer cell lines, MGC-803, SGC-7901 and BGC-823, as well as the normal gastric epithelial cell line, GES-1. (C) Correlation between the clinicopathological features and miR-150-5p expression in patients with GC. Data expressed as the mean \pm standard deviation. ${ }^{\# \#} \mathrm{P}<0.01$ vs. normal tissues; ${ }^{*} \mathrm{P}<0.05$ and ${ }^{* * *} \mathrm{P}<0.01$ vs. GES-1. miR, microRNA.

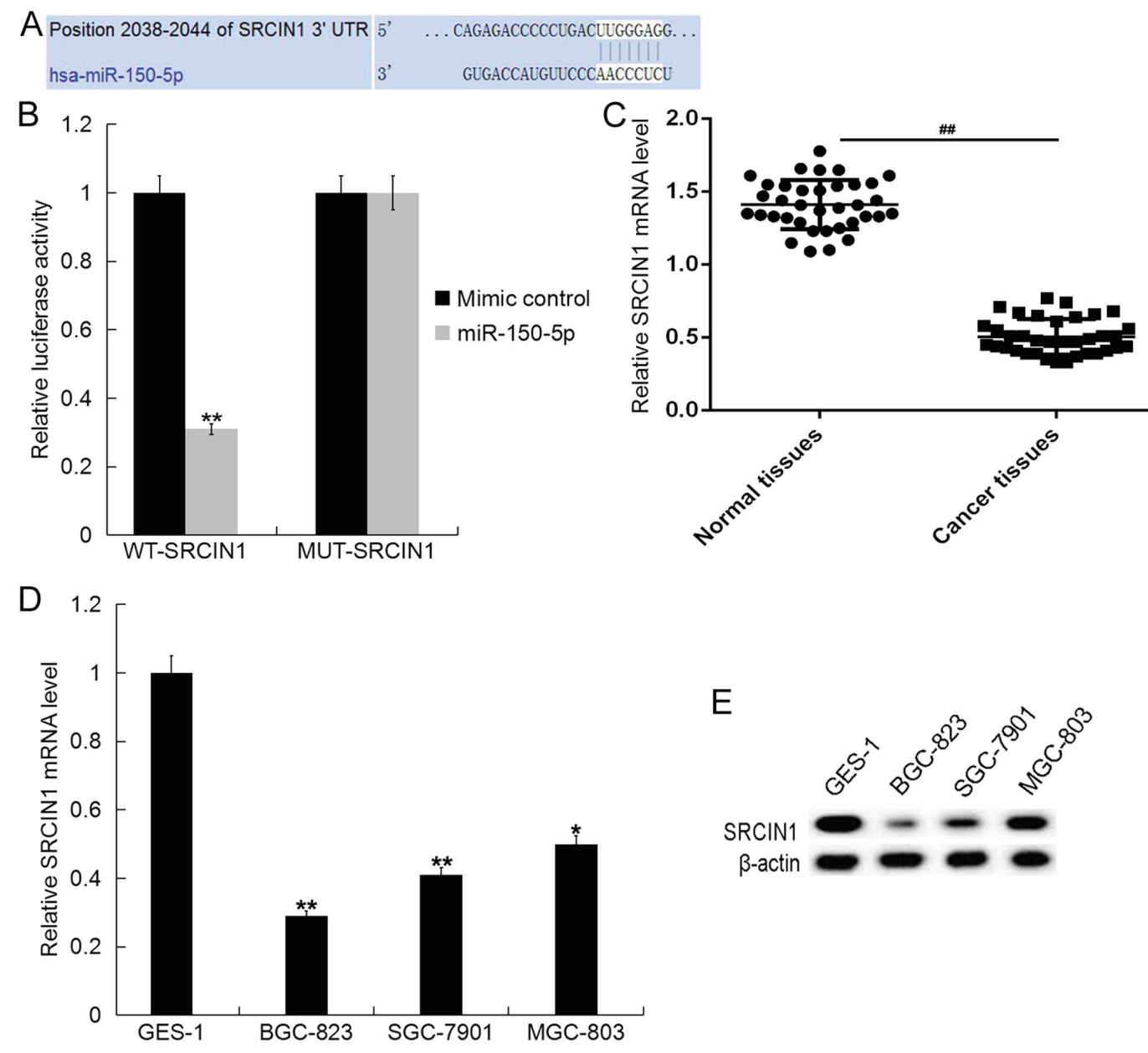

Figure 2. Association of miR-150-5p and SRCIN1. (A) The interaction between miR-150 and the 3'UTR of SRCIN1 was predicted using microRNA target site prediction software (TargetScan). (B) Luciferase activity of a reporter containing wild-type SRCIN1 3'UTR or mutant SRCIN1 3'UTR are presented. ${ }^{* *} \mathrm{P}<0.01$ vs. mimic control. reverse transcription-quantitative polymerase chain reaction was performed to detect SRCIN1 mRNA levels in (C) gastric cancer tissues and paired normal tissues, and in (D) gastric cancer cell lines (MGC-803, SGC-7901 and BGC-823) and normal gastric epithelial cells (GES-1). (E) Western blotting was performed to detect SRCIN1 protein levels in gastric cancer cell lines and normal gastric epithelial cells. All Data were presented as the mean \pm standard deviation of three independent experiments. ${ }^{* \prime \prime} \mathrm{P}<0.01$ vs. normal tissues; ${ }^{*} \mathrm{P}<0.05$ and ${ }^{* *} \mathrm{P}<0.01$ vs. GES-1. miR, microRNA, SRCIN1, SRC kinase signaling inhibitor 1; UTR, untranslated region.

miR-150-5p inhibitor suppresses GC cell viability and induces apoptosis. To assess the role of miR-150-5p in GC, a miR-150-5p inhibitor, SRCIN1-siRNA, or miR-150-5p
inhibitor+SRCIN1-siRNA was transfected into BGC-823 cells. Following 48-h transfection, transfection efficiency was detected using RT-qPCR and/or western blotting. The 
A

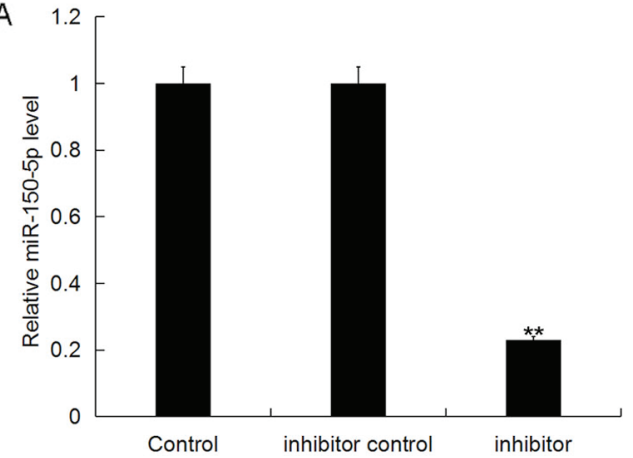

D

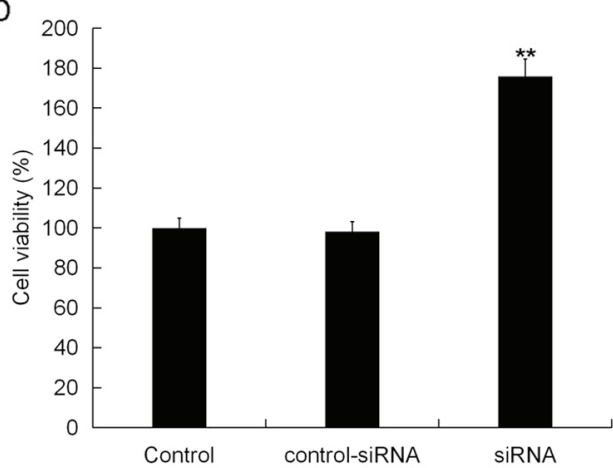

B

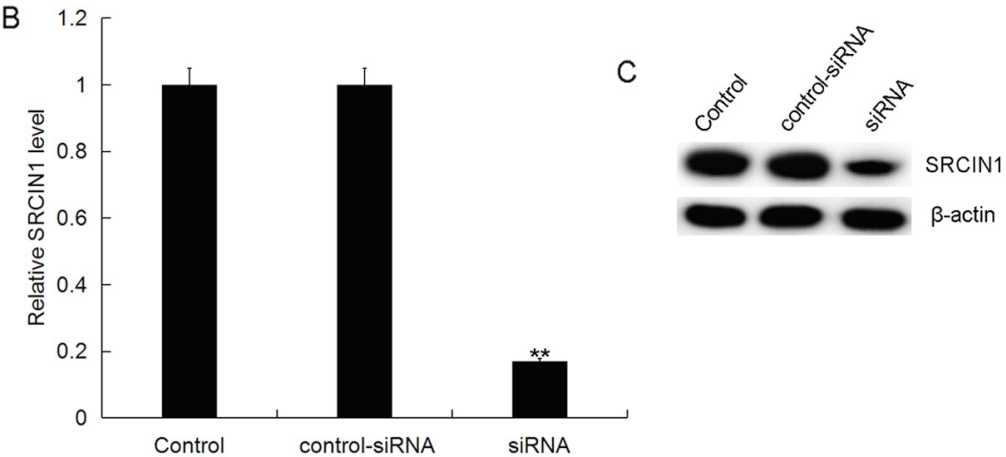

E
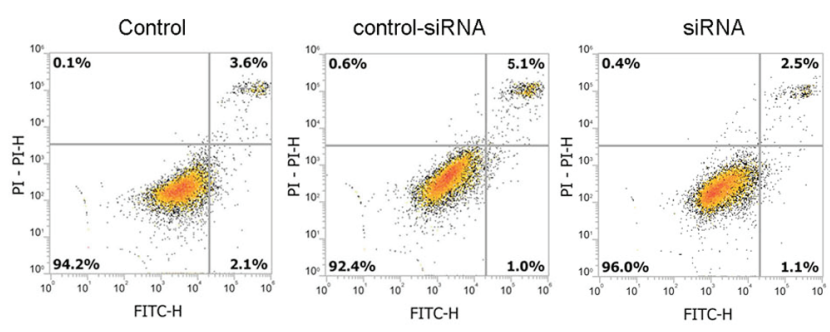

$\mathrm{F}$

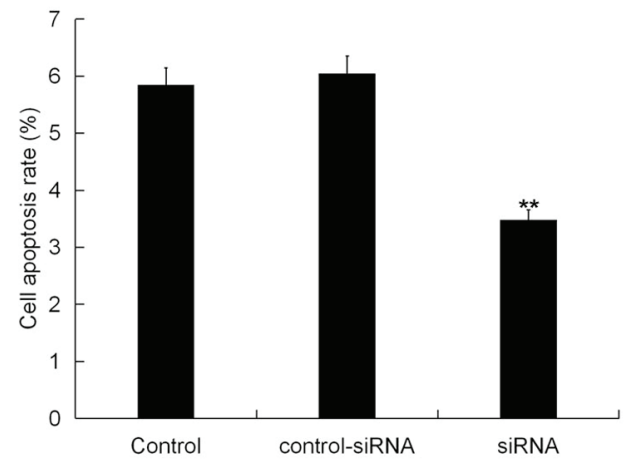

Figure 3. Effect of SRCIN1-siRNA on BGC-823 cell viability and apoptosis. (A) Following transfection with an inhibitor control or a miR-150-5p inhibitor, the level of miR-150-5p in BGC-823 cells was detected using RT-qPCR. Following transfection with control-siRNA or SRCIN1-siRNA, the (B) mRNA and (C) protein level of SRCIN1 in BGC-823 cells was detected using RT-qPCR and western blotting, respectively. (D) A cell counting kit-8 assay was used to detect the viability of cells in each group. (E) Flow cytometry and Annexin V-Fluorescein isothiocyanate/propidium iodide double staining were used to detect the apoptosis of cells in each group. (F) Cell apoptosis (early+late apoptosis=Q2+Q4) rate was calculated and presented. Data were presented as the mean \pm standard deviation. ${ }^{* *} \mathrm{P}<0.01$ vs. control. miR, microRNA; RT-qPCR, reverse transcription-quantitative polymerase chain reaction; SRCIN1, SRC kinase signaling inhibitor 1; siRNA, small interfering RNA.

results demonstrated that, compared with the control group, the miR-150-5p inhibitor significantly reduced miR-150-5p expression (Fig. 3A) and SRCIN1-siRNA significantly reduced the mRNA and protein level of SRCIN1 (Fig. 3B and C). The results also demonstrated that, compared with the control group, SRCIN1-siRNA significantly enhanced BGC-823 cell viability (Fig. 3D) and inhibited cell apoptosis (Fig. 3E and F), while control-siRNA had no effect on BGC-823 cell viability or apoptosis. These results suggest that miR-150-5p may be involved in regulating SRCIN1 expression in GC cells.

Additionally, compared with control group, the miR-150-5p inhibitor significantly increased SRCIN1 mRNA and protein expression, which was inhibited following SRCIN1-siRNA treatment (Fig. 4A and B). The results of the CCK-8 assay indicated that the miR-150-5p inhibitor decreased BGC-823 cell viability, whilst SRCIN1-siRNA treatment reversed this effect (Fig. 4C). Furthermore, it was revealed that the miR-150-5p inhibitor significantly induced BGC-823 cell apoptosis, which was reversed following SRCIN1-siRNA treatment (Fig. 4D and E).
miR-150-5p inhibitor suppresses EMT in BGC-823 cells. Finally, the current study assessed whether miR-150-5p affected GC cell EMT, interstitial cell markers (vimentin, $\mathrm{N}$-cadherin and $\beta$-catenin) and epithelial cell markers (ZO-1 and E-cadherin). The results indicated that compared with the control group, the miR-150-5p inhibitor reduced the expression of interstitial cell markers (vimentin, N-cadherin and $\beta$-catenin) and increased the expression of epithelial cell markers (ZO-1 and E-cadherin), thus inhibiting gastric cancer cell EMT. All these effects were reversed by SRCIN1-siRNA treatment (Fig. 5).

\section{Discussion}

GC is a malignant tumor that originates from the epithelium of gastric mucosa (1). The annual incidence of GC in China is $\sim 400,000$, which accounts for $42 \%$ of the total number of cases worldwide $(4,29)$. GC accounts for $10 \%$ of all diagnosed cancer types each year and accounts for $12 \%$ of 


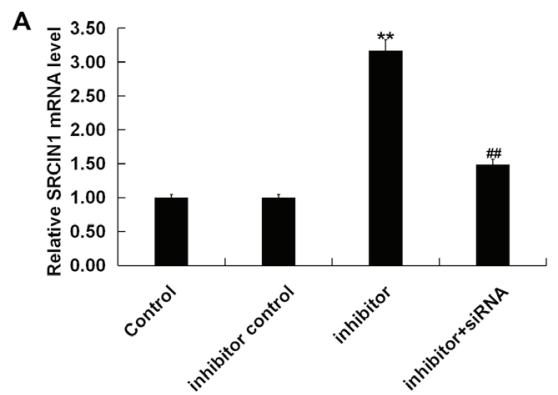

B

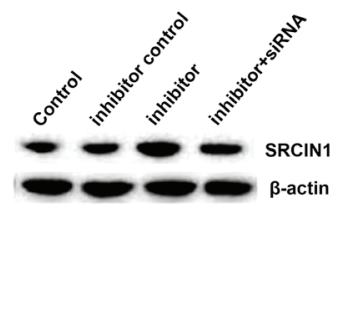

C

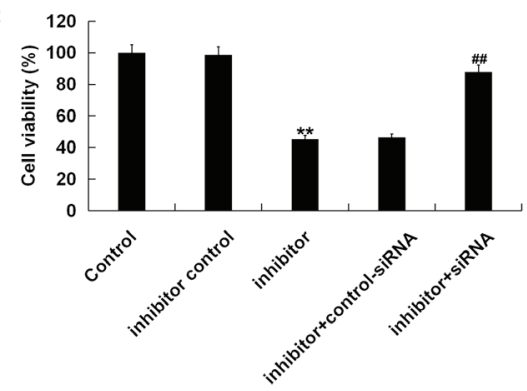

D

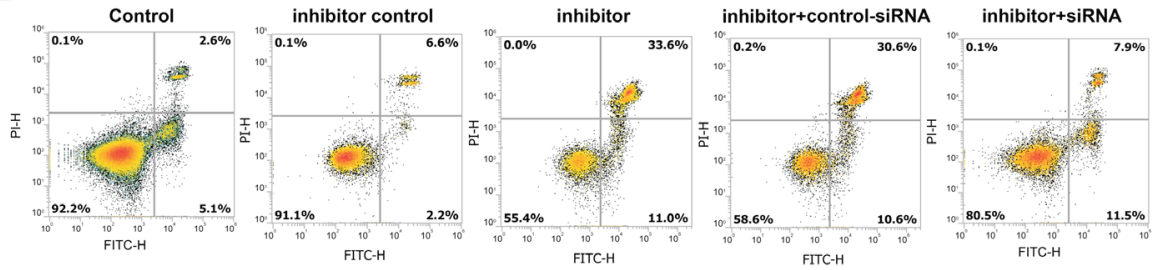

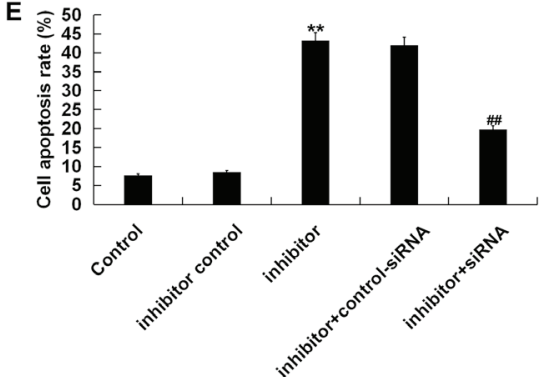

Figure 4. Effect of miR-150-5p on BGC-823 cell viability and apoptosis. Following transfection with an inhibitor control, a miR-150-5p inhibitor or an inhibitor+SRCIN1-siRNA, the (A) mRNA and (B) protein levels of SRCIN1 in BGC-823 cells were detected using RT-qPCR and western blotting, respectively. (C) A Cell Counting Kit-8 assay was used to detect the viability of cells in each group. (D) Flow cytometry and Annexin V-Fluorescein isothiocyanate/propidium iodide double staining were used to detect the apoptosis of cells in each group. (E) Cell apoptosis (early+late apoptosis=Q2+Q4) rate was calculated and presented. Data were presented as the mean \pm standard deviation. ${ }^{* * *} \mathrm{P}<0.01$ vs. control; ${ }^{\# \#} \mathrm{P}<0.01$ vs. inhibitor. miR, microRNA; RT-qPCR, reverse transcription-quantitative polymerase chain reaction; SRCIN1, SRC kinase signaling inhibitor 1; siRNA, small interfering RNA.

A

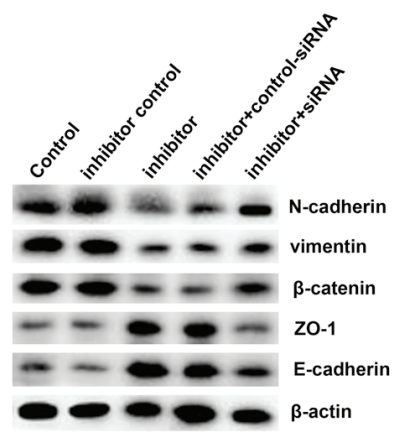

D

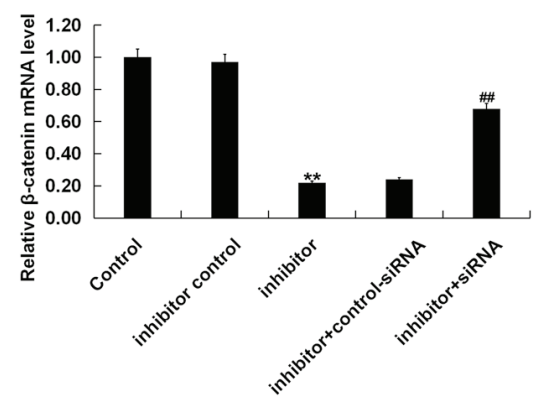

B

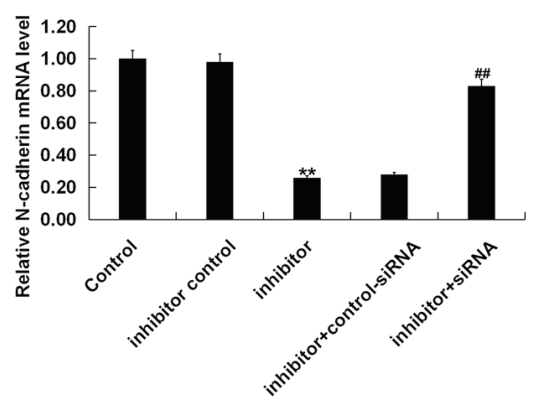

$\mathrm{E}$

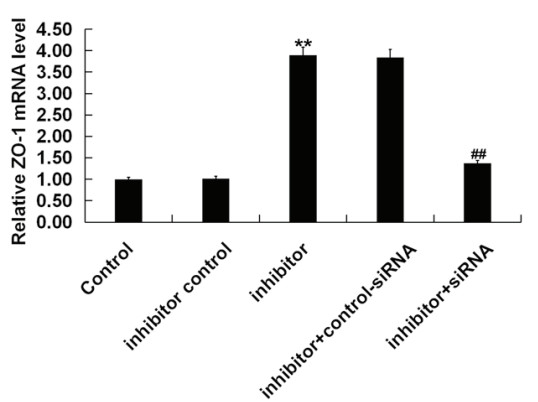

C
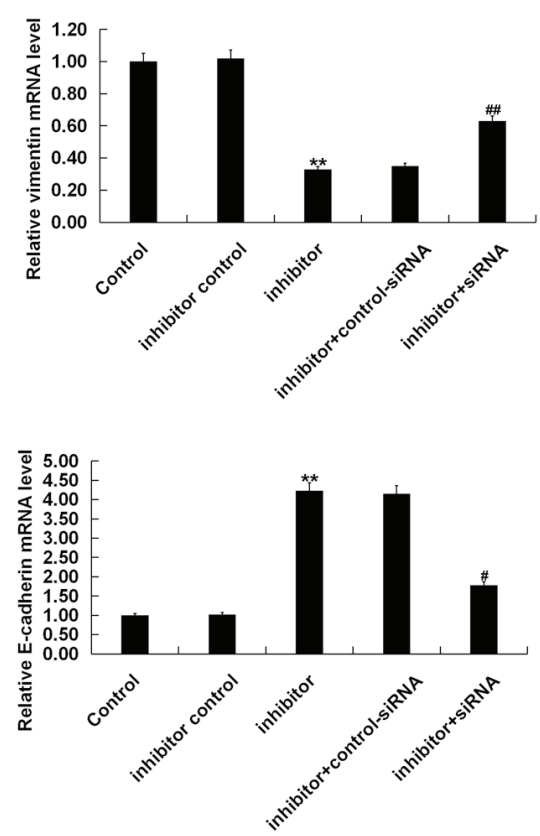

Figure 5. miR-150-5p inhibitor inhibits BGC-823 cell EMT. BGC-823 cells were transfected with an inhibitor control, a miR-150-5p inhibitor, and a miR-150-5p inhibitor+SRCIN1-siRNA for $48 \mathrm{~h}$. The (A) protein and mRNA expression of EMT factors including (B) N-cadherin, (C) vimentin and (D) $\beta$-catenin, and epithelial cell markers (E) ZO-1 and (F) E-cadherin were detected via western blotting and reverse transcription-quantitative polymerase chain reaction. Data were presented as the mean \pm standard deviation. ${ }^{* *} \mathrm{P}<0.01$ vs. control; ${ }^{*} \mathrm{P}<0.05$ and ${ }^{\# \#} \mathrm{P}<0.01$ vs. inhibitor. miR, microRNA, EMT, epithelial mesenchymal transition; SRCIN1, SRC kinase signaling inhibitor 1; siRNA, small interfering RNA; ZO-1, zonula occluden-1.

all cancer associated mortalities worldwide $(2,4)$. It is also one of the most common malignancies $(13,14)$. The current study assessed the role and mechanism of miR-150-SRCIN1 in the pathogenesis of GC development.
Many studies have determined the role of miRNAs in the development of various diseases (7,30-32) and further research has revealed that miRNAs are involved in the development of various types of cancer (33-36). Many 
studies have also identified the aberrant expression of certain miRNAs in tumor cells and thus are considered novel references for tumor diagnosis $(37,38)$. miRNAs are novel therapeutic targets and may serve as prognostic indicators (34). In recent years, a large number of studies have revealed that miRNAs serve a significant role in the development of GC $(39,40)$. However, to the best of our knowledge, the role of miR-150-5p in the pathological development of GC remains largely unclear. Therefore, the current study was performed.

The present study utilized RT-qPCR to detect the expression of miR-150-5p in different GC cell lines (including well-differentiated gastric adenocarcinoma MGC-803, moderately differentiated gastric adenocarcinoma SGC-7901 and poorly differentiated gastric adenocarcinoma BGC-823) and in the normal stomach mucosal epithelial cell line GES-1. The results revealed that miR-150-5p was significantly upregulated in all GC cell lines and the highest expression was exhibited in BGC-823 cells. Additionally, 36 GC tissue samples and adjacent normal tissues (including 12 cases of stage I GC, 12 cases of Stage II and 12 cases of Stage III-IV, with lymph node metastasis exhibited in 18 cases and no lymph metastasis in 18 cases) were utilized to assess the association between miR-150-5p expression and GC staging. The results revealed that the highest expression level of miR-150-5p was observed in GC patients at Stage III-IV, and the expression level of miR-150-5p was increased in GC patients with lymph node metastasis. This indicated that an increased expression of miR-150-5p was associated with advanced GC and lymph node metastasis.

The current study predicted that SRCIN1 was a target gene of miR-150-5p and this was confirmed by utilizing the dual luciferase reporter gene system. Lower expression levels of SRCIN1 were also demonstrated in GC tissues and cells, with the lowest expression exhibited in BGC-823 cells.

Furthermore, experiments were performed to assess the effects of miR-150-5p downregulation on BGC-823 cells, which exhibit the highest level of miR-150-5p among the GC cell lines examined. The results indicated that the miR-150-5p inhibitor decreased BGC-823 cell viability and induced cell apoptosis.

EMT is an important process of cancer cell migration and metastasis that can be induced by various transcription factors and signal transduction factors $(21,22,24)$. miRNAs, as small RNAs that regulate protein translation at the post-transcriptional level, are also involved in the EMT process. The present study assessed the effects of miR-150-5p treatment on BGC-823 cell EMT. The results revealed that the expression of vimentin, $\mathrm{N}$-cadherin and $\beta$-catenin were significantly decreased, while the expression of E-cadherin and ZO-1 were increased in BGC-823 cells transfected with the miR-150-5p inhibitor, indicating an inhibitory effect on GC cell EMT. In addition, the current study determined that all the effects of the miR-150-5p inhibitor on BGC-823 cells were reversed following SRCIN1-siRNA treatment. Furthermore, the results indicated that control-siRNA exhibited no significant effect on SRCIN1 expression at the mRNA or protein level. Therefore, an inhibitor + control-siRNA group was not included in the present study.
In conclusion, the results of the current study revealed that the miR-150-5p inhibitor inhibited GC cell viability, induced cell apoptosis and inhibited GC cell EMT by targeting SRCIN1. Therefore, the miR-150-5p/SRCIN1 axis may be considered as a potential clinical indicator and therapeutic target for the treatment of GC. However, the current study only performed a preliminary assessment of the role of miR-150-5p in GC. To validate these results, further experimental research is required. For example, the protein level of SRCIN1 in GC tissues should be determined and the correlation between SRCIN1 and miR-150-5p should be examined. In addition, the association between the expression levels of SRCIN1 or miR-150-5p with the clinicopathological features of patients with GC should be assessed. The current study aims to determine these in the future.

\section{Acknowledgements}

The authors would like to thank Dr Anlie Cai and Dr Huaide Peng, Zhuzhou Central Hospital for their support for experimental funding. The authors would like to thank Professor Jiansi Zhu, Cancer Research Institute of Nanhua University for his guidance on the experimental methods used in the current study, and Professor Zhijiao Zhou, Department of Pathology, Third Xiangya Hospital, for the help he provided during the preparation of the manuscript.

\section{Funding}

No funding received.

\section{Availability of data and materials}

The analyzed datasets used and/or generated during the present study are available from the corresponding author on reasonable request.

\section{Authors' contributions}

XQ contributed to data collection, statistical analysis, data interpretation and manuscript preparation. DC contributed to the study design and literature search. ML contributed to the study design, statistical analysis and data interpretation; $\mathrm{XC}$ contributed to statistical analysis and literature search; $\mathrm{MH}$ contributed to data collection and data interpretation. All authors read and approved the final manuscript.

\section{Ethics approval and consent to participate}

The present study was approved by the Ethical Committee of the Zhuzhou Central Hospital and written informed consent was obtained from each patient.

\section{Patient consent for publication}

Not applicable.

\section{Competing interests}

The authors declare that they have no competing interests. 


\section{References}

1. Yu ZY, Wang Z, Lee KY, Yuan P and Ding J: Effect of silencing colon cancer-associated transcript 2 on the proliferation, apoptosis and autophagy of gastric cancer BGC-823 cells. Oncol Lett 15: 3127-3132, 2018 .

2. Salem M, Xiu J, Eldeiry W, Reddy S, Philip P, Gatalica Z, Khan S, Denlinger C, Mikhail S, Smaglo B, et al: Comparative molecular analyses of esophageal adenocarcinoma, esophageal squamous cell carcinoma and gastric adenocarcinoma, and impact of molecular profile on outcome. Ann Oncol 27: ii119-ii119, 2016.

3. Fu L, Yin F, Li XR, Han BK, Zhang C, Wang JW, Wang YQ, Bi YF and Liu HM: Generation and characterization of a paclitaxel-resistant human gastric carcinoma cell line. Anticancer Drugs 1: 491-502, 2018.

4. Yang BF, Cai W and Chen B: LncRNA SNHG12 regulated the proliferation of gastric carcinoma cell BGC-823 by targeting microRNA-199a/b-5p. Eur Rev Med Pharmacol Sci 22 1297-1306, 2018

5. Liu L, Si N, Ma Y, Ge D, Yu X, Fan A, Wang X, Hu J, Wei P, Ma L, et al: Hydroxysafflor-yellow A induces human gastric carcinoma BGC-823 cell apoptosis by activating peroxisome proliferator-activated receptor gamma (PPAR $\gamma)$. Med Sci Monit 24: 803-811, 2018.

6. Rothmiller S, Wolf M, Worek F, Steinritz D, Thiermann H and Schmidt A: Alteration of miRNA expression in a sulfur mustard resistant cell line. Toxicol Lett 293: 38-44, 2018.

7. Ultimo S, Martelli AM, Zauli G, Vitale M, Calin GA and Neri LM: Roles and clinical implications of MicroRNAs in acute lymphoblastic leukemia. J Cell Physiol 233: 5642-5654, 2018

8. Sheng N, Zhang L and Yang S: MicroRNA-429 decreases the invasion ability of gastric cancer cell line BGC-823 by downregulating the expression of heparanase. Exp Ther Med 15: 1927-1933, 2018.

9. Meng Y, Tian H, Hu Q, Liang H, Zeng L and Xiao H: MicroRNA repertoire and comparative analysis of Andrias davidianus infected with ranavirus using deep sequencing. Dev Comp Immunol 85: 108-114, 2018.

10. Yan J,Li X, Peng L, Shen X, Dang Y and Zhang G: MicroRNA-150 as a potential biomarker in diagnosis of cancer: A meta-analysis. Clin Lab 63: 1187-1197, 2017.

11. Li C, Du X, Xia S and Chen L: MicroRNA-150 inhibits the proliferation and metastasis potential of colorectal cancer cells by targeting iASPP. Oncol Rep 40: 252-260, 2018.

12. Tang W, Xu P, Wang H, Niu Z, Zhu D, Lin Q, Tang L and Ren L: MicroRNA-150 suppresses triple-negative breast cancer metastasis through targeting HMGA2. Onco Targets Ther 11 : 2319-2332, 2018

13. Sun X, Zhang C, Cao Y and Liu E: miR-150 suppresses tumor growth in melanoma through downregulation of MYB. Oncol Res 27: 317-323, 2019.

14. Zhang Z, Wang J, Li J, Wang X and Song W: MicroRNA-150 promotes cell proliferation, migration and invasion of cervical cancer through targeting PDCD4. Biomed Pharmacother 97: 511-517, 2018.

15. Koshizuka K, Hanazawa T, Kikkawa N, Katada K, Okato A, Arai T, Idichi T, Osako Y, Okamoto Y and Seki N: Antitumor miR-150-5p and miR-150-3p, inhibit cancer cell aggressiveness by targeting SPOCK1, in head and neck squamous cell carcinoma. Auris Nasus Larynx 45: 854-865, 2018.

16. Yan R, Yang T,Zhai H,Zhou Z, GaoL andLi Y: MicroRNA-150-5p affects cell proliferation, apoptosis and EMT by regulation of the BRAFV600E mutation in papillary thyroid cancer cells. J Cell Biochem 119: 8763-8772, 2018.

17. Ergun S, Güney S, Temiz E, Petrovic N and Gunes S: Significance of Mir-15a-5p and Cnksr3 as novel prognostic biomarkers in non-small cell lung cancer. Anticancer Agents Med Chem 18 1695-1701, 2018.

18. Yu J, Feng Y, Wang Y and An R: Aryl hydrocarbon receptor enhances the expression of miR-150-5p to suppress in prostate cancer progression by regulating MAP3K12. Arch Biochem Biophys 654: 47-54, 2018

19. Suetsugu T, Koshizuka K, Seki N, Mizuno K, Okato A, Arai T, Misono S, Uchida A, Kumamoto T and Inoue H: Downregulation of matrix metalloproteinase 14 by the antitumor miRNA miR-150-5p, inhibits the aggressiveness of lung squamous cell carcinoma cells. Int J Oncol 52: 913-924, 2018.
20. Wang S, Yan Y, Cheng Z, Hu Y and Liu T: Sotetsuflavone suppresses invasion and metastasis in non-small-cell lung cancer A549 cells by reversing EMT via the TNF- $\alpha / N F-\kappa B$ and PI3K/AKT signaling pathway. Cell Death Discov 4: 26 , 2018.

21. Zuo L, Zhao H, Yang R, Wang L, Ma H, Xu X, Zhou P and Kong L: Lamin A/C might be involved in the EMT signalling pathway. Gene 663: 51-64, 2018.

22. Yang Y, Gao M, Lin Z, Chen L, Jin Y, Zhu G, Wang Y and Jin T: DEK promoted EMT and angiogenesis through regulating $\mathrm{PI} 3 \mathrm{~K} / \mathrm{AKT} / \mathrm{mTOR}$ pathway in triple-negative breast cancer. Oncotarget 8: 98708-98722, 2017.

23. Pal M, Bhattacharya S, Kalyan G and Hazra S: Cadherin profiling for therapeutic interventions in Epithelial Mesenchymal Transition (EMT) and tumorigenesis. Exp Cell Res 368: 137-146, 2018.

24. Ma Z, Xin Z, Hu W, Jiang S, Yang Z, Yan X, Li X, Yang Y and Chen F: Forkhead box O proteins: Crucial regulators of cancer EMT. Semin Cancer Biol 50: 21-31, 2018

25. Liu CY, Lin HH, Tang MJ and Wang YK: Vimentin contributes to epithelial-mesenchymal transition cancer cell mechanics by mediating cytoskeletal organization and focal adhesion maturation. Oncotarget 6: 15966-15983, 2015.

26. Shang $\mathrm{S}$, Hua $\mathrm{F}$ and $\mathrm{Hu} \mathrm{ZW}$ : The regulation of $\beta$-catenin activity and function in cancer: Therapeutic opportunities. Oncotarget 8 : 33972-33989, 2017.

27. Zihni C, Mills C, Matter K and Balda MS: Tight junctions: From simple barriers to multifunctional molecular gates. Nat Rev Mol Cell Biol 17: 564-580, 2016.

28. Livak KJ and Schmittgen TD: Analysis of relative gene expression data using real-time quantitative PCR and the 2(-Delta Delta C(T)) method. Methods 25: 402-408, 2001.

29. Ferlay J, Soerjomataram I, Dikshit R, Eser S, Mathers C, Rebelo M, Parkin DM, Forman D and Bray F: Cancer incidence and mortality worldwide: Sources, methods and major patterns in GLOBOCAN 2012. Int J Cancer 136: E359-E386, 2015.

30. Mellis D and Caporali A: MicroRNA-based therapeutics in cardiovascular disease: Screening and delivery to the target. Biochem Soc Trans 46: 11-21, 2018.

31. Cao DD, Li L and Chan WY: MicroRNAs: Key regulators in the central nervous system and their implication in neurological diseases. Int J Mol Sci 17: pii: E842, 2016

32. Mannucci C, Casciaro M, Minciullo PL, Calapai G, Navarra M and Gangemi S: Involvement of microRNAs in skin disorders: A literature review. Allergy Asthma Proc 38: 9-15, 2017.

33. Hu L, Ai J, Long H, Liu W, Wang X, Zuo Y, Li Y, Wu Q and Deng Y: Intergrative microRNA and gene profiling data analysis reveals novel biomarkers and mechanisms for lung cancer. Oncotarget 7: 8441-8454, 2016

34. Tsai MM, Wang CS, Tsai CY, Huang HW, Chi HC, Lin YH, Lu PH and Lin KH: Potential diagnostic, prognostic and therapeutic targets of microRNAs in human gastric cancer. Int $\mathrm{J}$ Mol Sci 17: pii: E945, 2016.

35. Zhao H, Li M, Li L, Yang X, Lan G and Zhang Y: MiR-133b is down-regulated in human osteosarcoma and inhibits osteosarcoma cells proliferation, migration and invasion and promotes apoptosis. PLoS One 8: e83571, 2013.

36. Kaukoniemi KM, Rauhala HE, Scaravilli M, Latonen L, Annala M, Vessella RL, Nykter M, Tammela TL and Visakorpi T: Epigenetically altered miR-193b targets cyclin D1 in prostate cancer. Cancer Med 4: 1417-1425, 2015.

37. Schmidt A, Steinritz D, Thiermann H, Meineke V and Abend M: Alteration of miRNA expression in early endothelial cells after exposure with sub-lethal sulfur mustard concentrations. Toxicol Lett 244: 88-94, 2016.

38. Gharbi S, Khateri S, Soroush MR, Shamsara M, Naeli P, Najafi A, Korsching E and Mowla SJ: MicroRNA expression in serum samples of sulfur mustard veterans as a diagnostic gateway to improve care. PLoS One 13: e0194530, 2018.

39. Ma M, Zhao J, Wu Q, Xiao K, Li S, Zhu H, Liu C, Xie H and Zuo C: MiRNA-545 negatively regulates the oncogenic activity of EMS1 in gastric cancer. Cancer Med 7: 2452-2462, 2018.

40. Liao Y, Liao Y, Li J, Xiao K, Li S, Zhu H, Liu C, Xie H and Zuo C: Genetic variants in miRNA machinery genes associated with clinicopathological characteristics and outcomes of gastric cancer patients. Int J Biol Markers 33: 301-307, 2018. 